\title{
Decaying hadrons within constituent-quark models
}

\author{
R. Kleinhappel* and W. Schweiger ${ }^{\dagger}$ \\ Institut für Physik, Universität Graz, A-8010 Graz, Austria \\ E-mail: regina.kleinhappelduni-graz.at \\ wolfgang.schweigerduni-graz.at
}

Within conventional constituent-quark models hadrons come out as stable bound states of the valence (anti)quarks. Thereby the resonance character of hadronic excitations is completely ignored. A more realistic description of hadron spectra can be achieved by including explicit mesonic degrees of freedom, which couple directly to the constituent quarks. We will present a coupledchannel formalism that describes such hybrid systems in a relativistically invariant way and allows for the decay of excited hadrons. The formalism is based on the point-form of relativistic quantum mechanics. If the confining forces between the (anti)quarks are described by instantaneous interactions it can be formally shown that the mass-eigenvalue problem for a system that consists of dynamical (anti)quarks and mesons reduces to a hadronic eigenvalue problem in which the eigenstates of the pure confinement problem (bare hadrons) are coupled via meson loops. The only point where the quark substructure enters are form factors at the meson-(bare) hadron vertices. The physical picture that emerges resembles the kind of hadronic resonance model that has been developed by Sato and Lee and is now heavily used at the Excited Baryon Analysis Center (EBAC) to fix $N^{*}$ properties. Our approach, however, is in a certain sense inverse to the one of Sato and Lee. Whereas they want to undress physical resonances to end up with bare quantities, we rather want to dress the bound-states resulting from a pure constituent quark model to end up with quantities that can be directly compared with experiment. The way how our approach works will be exemplified by means of a simple quark-antiquark-meson system.

Sixth International Conference on Quarks and Nuclear Physics,

April 16-20, 2012

Ecole Polytechnique, Palaiseau, Paris

*Supported by Fonds zur Förderung der wissenschaftlichen Forschung in Österreich (FWF DK W1203-N16)

$\dagger$ Speaker. 


\section{Introduction}

Although conventional constituent-quark models, in particular their relativistic versions, are very successful in reproducing masses and electroweak properties of the hadron ground states and of the lowest excited states (see Refs. [ [U, [1, []] and references therein), they are obviously lacking an important piece of physics. Hadronic excitations result as infinitely long-lived bound states of the valence (anti)quarks and not as decaying resonances with a finite lifetime. Strong decays of hadronic resonances are then usually treated in leading-order perturbation theory, assuming a particular form for the elementary decay vertex (e.g. ${ }^{3} P_{0}$-model [四] or elementary emission model [5]) and taking the bound-state wave functions as resulting from the favorite constituent-quark model for the incoming and outgoing hadronic states. But it turns out that a large portion of the calculated partial decay widths underestimate the experimental values by at least one order of magnitude [ [2, []]. Nevertheless, one can observe a certain pattern in the decay widths which may be used as an additional tool for classifying hadronic states according to $S U(3)$-flavor multiplets [罒].

A good starting point for a more realistic description of hadron resonances is the physical picture that hadrons consist of a quark core which is surrounded by a meson cloud. A simple way to realize this kind of picture within a constituent-quark model is to introduce, in addition to the valence-(anti)quark degrees-of-freedom, mesons that can be emitted and absorbed by the (anti)quarks. Efforts in this direction have been made already (see Secs. 2.1 and 4.1 of Ref. [6] for an overview), but mostly within non-relativistic settings. This is certainly inadequate for hadrons consisting of light quarks as, e.g., calculations of the electroweak structure of hadrons clearly revealed [ $[\mathbb{8}]$. A Poincaré invariant formalism for the description of hybrid systems consisting of valence (anti)quarks and (elementary) mesons has been proposed in Ref. [U], where this formalism was applied to the calculation of vector-meson masses. In the present work we will follow this approach. It is based on the point-form of relativistic quantum mechanics and makes use of the Bakamjian-Thomas construction [0] to guarantee Poincaré invariance. But in contrast to Ref. [四] we will also allow for absorption of the emitted meson by the emitting (anti)quark and not only for the exchange of the meson between different hadronic constituents.

The relativistic multichannel formalism which we use to treat such hybrid models that incorporate valence (anti)quarks and mesons is sketched in Sec. 2. We will show, under the assumption of instantaneous confining forces, that the corresponding mass-eigenvalue problem can be equivalently reformulated as a mass-eigenvalue problem on the hadronic level, in which bare hadrons are mixed via meson loops. Sec. 3 is devoted to a numerical study of a very simple system consisting of a quark-antiquark pair and a meson, all treated as scalar particle. This section contains also our conclusions and an outlook.

\section{Relativistic coupled-channel framework}

The point-form version of the the Bakamjian-Thomas construction amounts to the assumption that the (interacting) 4-momentum operator can be factorized into a free 4-velocity operator and an interaction-dependent mass operator

$$
\hat{P}^{\mu}=\hat{M} \hat{V}_{\text {free }}^{\mu}=\left(\hat{M}_{\text {free }}+\hat{M}_{\text {int }}\right) \hat{V}_{\text {free }}^{\mu} .
$$


Poincaré invariance holds if the interaction term $\hat{M}_{\text {int }}$ is a Lorentz scalar and commutes with $\hat{V}_{\text {free }}^{\mu}$. Equation (R. لل implies that the overall velocity of the system can be easily separated from the internal motion which is described by the mass operator $\hat{M}$. This separation is most conveniently done by representing the operators in terms of velocity states which specify the state of the system through its overall velocity and the CM-momenta of its components [ए]].

Treating the emission and absorption of particles within such a quantum-mechanical framework requires a multichannel formulation. In the simplest case of only 1 meson $M$ that can be emitted and absorbed by the valence (anti)quarks one has to deal with a 2-channel problem. The corresponding mass-eigenvalue equation reads

$$
\left(\begin{array}{cc}
M_{\mathrm{val}} & K \\
K^{\dagger} & M_{\mathrm{val}, M}
\end{array}\right)\left(\begin{array}{l}
\left|\Psi_{\mathrm{val}}\right\rangle \\
\left|\Psi_{\mathrm{val}, M}\right\rangle
\end{array}\right)=m\left(\begin{array}{l}
\left|\Psi_{\mathrm{val}}\right\rangle \\
\left|\Psi_{\mathrm{val}, M}\right\rangle
\end{array}\right) .
$$

$K^{(\dagger)}$ is a vertex operator that accounts for the absorption (emission) of the meson $M$ by a quark or antiquark. Its velocity-state matrix elements are obtained from the corresponding quantum-field theoretical interaction Lagrangian density []]. The diagonal terms $M_{\mathrm{val}}$ and $M_{\mathrm{val}, M}$ of the matrix mass operator $\hat{M}$ contain, in addition to the relativistic kinetic energies of the valence (anti)quarks and the meson $M$, an instantaneous confining potential between the (anti)quarks and, possibly, also an instantaneous hyperfine interaction. As it turns out in the following, the assumption that confinement is instantaneous will be crucial for the physical interpretation of this kind of model. Physical hadrons, i.e. eigenstates of $\hat{M}$, are then a superposition of $\left|\Psi_{\text {val }}\right\rangle$ and $\left|\Psi_{\text {val }, M}\right\rangle$. In order to solve the mass-eigenvalue equation (2.2) we, however, eliminate $\left|\Psi_{\mathrm{val}, M}\right\rangle$ to end up with an equation for $\left|\Psi_{\text {val }}\right\rangle$ alone:

$$
(M_{\mathrm{val}}+\underbrace{K\left(m-M_{\mathrm{val}, M}\right)^{-1} K^{\dagger}}_{=V_{\mathrm{opt}}(m)})\left|\Psi_{\mathrm{val}}\right\rangle=m\left|\Psi_{\mathrm{val}}\right\rangle .
$$

$V_{\text {opt }}$ is an optical potential that describes the emission of the meson $M$ by a valence (anti)quark and the subsequent absorption by another or the same valence (anti)quark. The important point to note here is that the system which propagates in the intermediate state consists of a meson and confined valence (anti)quarks. Therefore the valence (anti)quarks are not renormalized by meson loops. Equation ([2.3) can now be further reduced to an algebraic equation by expanding $\left|\Psi_{\text {val }}\right\rangle$ in terms of eigenstates of $M_{\mathrm{val}}$ :

$$
\left|\psi_{\text {val }}\right\rangle=\sum_{n} A_{n}|n\rangle \quad \text { with } \quad M_{\text {val }}|n\rangle=\mu_{n}|n\rangle .
$$

We will call the eigenstates $|n\rangle$ of $M_{\mathrm{val}}$ "bare hadrons". A physical hadron is thus a superposition of bare hadrons with the coefficients $A_{n}$ being given by the set of algebraic equations

$$
\left(m-\mu_{n}\right) A_{n}=\sum_{n^{\prime}}\left\langle n\left|V_{\mathrm{opt}}(m+i \varepsilon)\right| n^{\prime}\right\rangle A_{n^{\prime}}, \quad n=0,1,2,3, \ldots
$$

This system of equations is now already an eigenvalue problem on the hadronic level that describes bare hadrons which are coupled via meson loops. A further analysis of the matrix elements of the optical potential reveals indeed that all the valence-(anti)quark substructure of the bare hadrons 


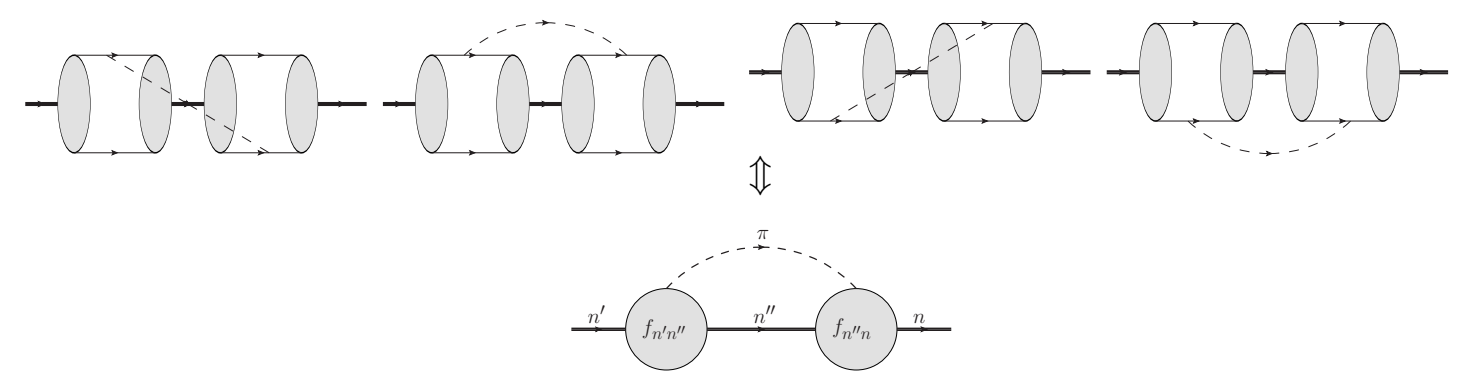

Figure 1: Graphical representation of the optical potential showing up in Eq. (2.5) for a confined quarkantiquark system that is allowed to emit and reabsorb a pion. The optical potential on the constituent level is represented by the upper graphs, the equivalent optical potential on the hadronic level by the lower graph.

can be absorbed into strong meson-(bare) hadron vertex form factors $f_{n, n^{\prime}}\left(\left|\vec{k}_{M}\right|\right)$ (see Fig. W) such that our model acquires an equivalent formulation on the hadronic level. It is now important to note that the optical potential becomes singular as soon as $m>\mu_{0}+m_{M}$, i.e. the mass eigenvalue $m$ becomes larger than the mass $\mu_{0}$ of the bare ground state $|n=0\rangle$ plus the meson mass $m_{M}$. This singularities are treated by means of the usual $i \varepsilon$-prescription. As a consequence, the matrix elements of the optical potential $\left\langle n\left|V_{\text {opt }}(m+i \varepsilon)\right| n^{\prime}\right\rangle$ become complex. Most importantly, this leads to complex mass eigenvalues $\mathbf{m}_{j}=m_{j}+i \Gamma_{j} / 2$ and thus to finite decay widths $\Gamma_{j}$ for excited states $(j>0)$. Practically we have solved the eigenvalue equation (2.5) in which the mass eigenvalue $m$ enters in a non-linear way (via $V_{\mathrm{opt}}(m)$ ) by means of an iterative solution method which provides also the complex mass eigenvalues [12]].

\section{Numerical results and outlook}

First numerical studies along these lines were performed in Ref. [12] for a simple model of meson resonances. The degrees-of-freedom of this model are 1 quark, 1 antiquark and 1 pion that can be emitted and absorbed by the (anti)quark. Spin and flavor are neglected in this model and confinement of the quark-antiquark pair is described via a harmonic-oscillator potential (added, however, to $M_{q \bar{q}, \text { free }}^{2}$ ). The mass parameters of this model were prefixed to commonly used values $\left(m_{q}=m_{\bar{q}}=0.34 \mathrm{GeV}, m_{\pi}=0.135 \mathrm{GeV}\right)$. The $\pi-q$ coupling constant $g$ was varied within a reasonable range to study its effect on the mass eigenvalues and the decay widths. To give the model some physical meaning the parameters of the confinement potential (oscillator strength $a=0.28 \mathrm{GeV}$ and parameter $V_{0}=0.1 \mathrm{GeV}$ that fixes the ground-state mass) were determined a posteriori such that the $\omega(782)$ and $\omega(1420)$ masses are approximately reproduced for the maximum decay width $\Gamma_{1}$ that can be achieved. The study was restricted to $s$-wave mesons and orbital excitations were neglected in intermediate states.

The first physical information one can extract from this model is the structure of the pion(bare)-meson vertices which is encoded in the (strong) form factors $f_{n, n^{\prime}}\left(\left|\vec{k}_{\pi}\right|\right)$. Two of these form factors are plotted in Fig. $\square$. The dependence of the ground-state mass and the mass and width of the first excited state on the $\pi-q$ coupling constant $g$ is shown in Fig. [1]. The $\pi$-loop obviously provides an attractive force which pushes the masses downwards. The decay width of the first excited state $\Gamma_{1}$ exhibits a maximum as function of $g$ and vanishes when $\operatorname{Re}\left(\mathbf{m}_{1}\right)=\mu_{0}+m_{\pi}$. This behavior 

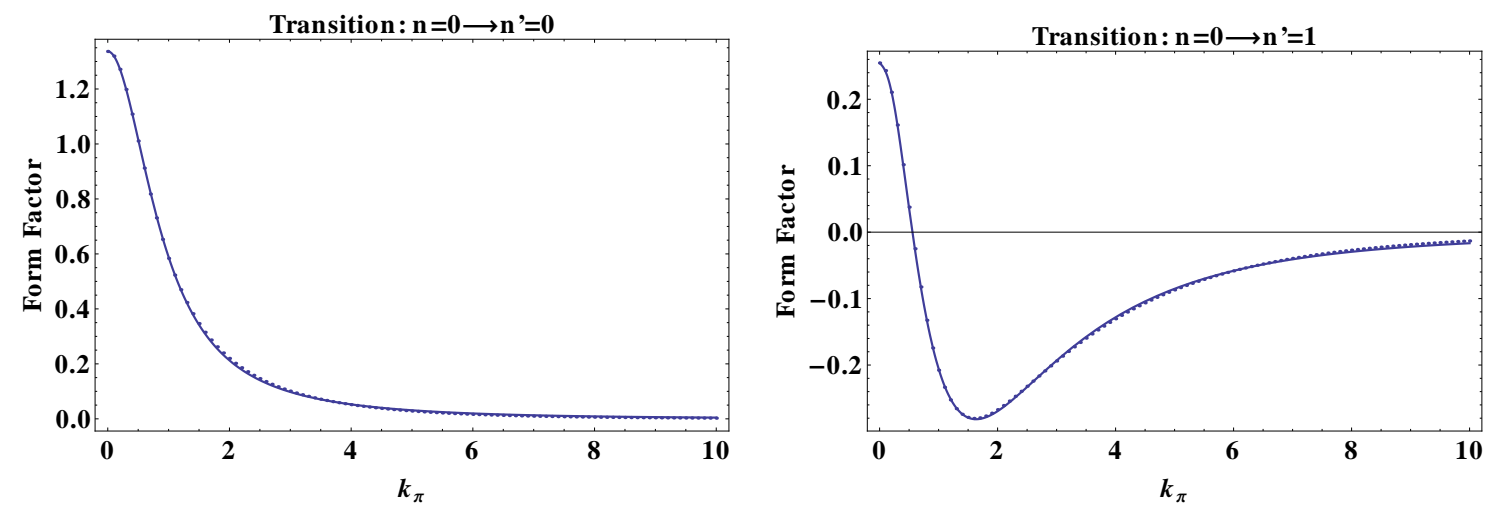

Figure 2: Left: elastic form factor for the coupling of a pion to the harmonic-oscillator ground state $(n=$ $n^{\prime}=0$ ). Right: transition form factor associated with pion emission causing a transition of the $n=0$ to the $n^{\prime}=1$ harmonic oscillator state. Form factors are plotted as functions of $\left|\vec{k}_{\pi}\right|$ and are not normalized.

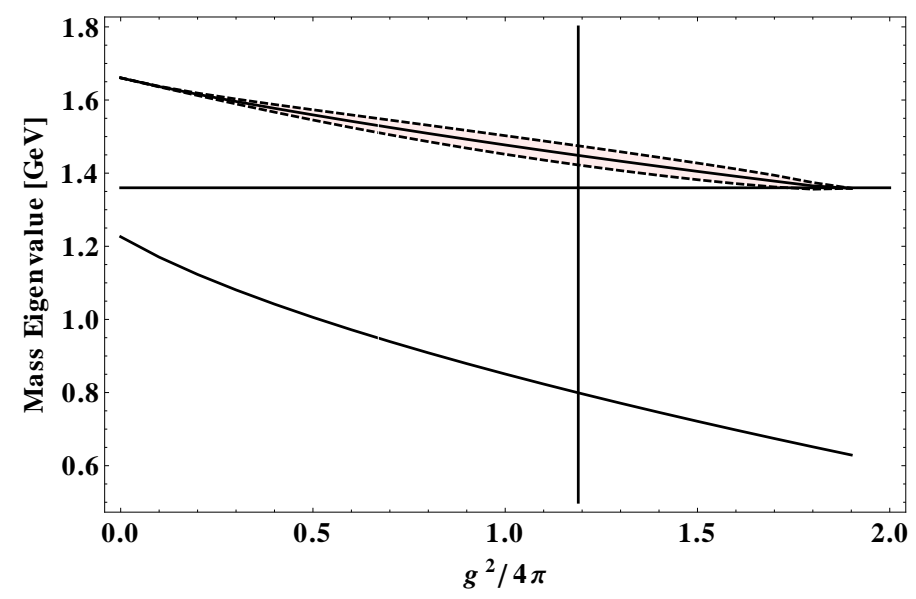

Figure 3: Dependence of the ground state and the first excited state on the $\pi$ - $q$ coupling constant $g$. The shaded band indicates the decay width $\Gamma_{1}$ of the first excited state (multiplied by 4 for better visibility). The positions of the maximal decay width $\left(\Gamma_{1}=0.026 \mathrm{GeV}\right.$ reached at $\left.g^{2} / 4 \pi=1.19 \mathrm{GeV}^{2}\right)$ and of the decay threshold of the first excited state $\left(\right.$ at $\mu_{0}+m_{\pi}$ ) are indicated by vertical and horizontal lines, respectively.

indicates that our simple model, although it provides hadron excitations with finite lifetimes, is still not complete. Instead of decaying into the physical ground state (mass $\mathbf{m}_{0}$ ) and a $\pi$ the first excited state can only decay into the bare ground state (mass $\mu_{0}$ ) and a $\pi$. A way out would be to increase the number of channels and include $q \bar{q} 2 \pi, q \bar{q} 3 \pi$, etc. channels. This has do be done until the probability of finding $n$ pions in the (physical) meson becomes negligibly small. The technical problems associated with such a procedure would soon become rather big, maybe even unsurmountable.

Another, more promising strategy would be to consider the present calculation just as a first step to "dress" bare hadrons (i.e. the confined valence-(anti)quark states) by means of meson loops. The important point to notice is that in our approach this dressing happens already on the hadronic level and not on the quark level if confinement is represented by an instantaneous interaction. In 
a next step the meson-(bare)hadron vertices and the masses of the bare hadrons, coming from the hybrid quark model, could be used as the basic input for a dynamical coupled-channel calculation of hadron masses and decay widths à la Sato and Lee [13]]. In this second step all the calculations are already done on the hadronic level. Put into action, such a bottom-up approach could provide an understanding of hadron resonances in terms of constituent-quark degrees-of-freedom that goes beyond pure hadron reaction models à la Sato and Lee and it would substantiate the physical picture of hadrons consisting of a quark core that is surrounded by a meson cloud.

\section{References}

[1] S. Capstick and W. Roberts, Prog. Part. Nucl. Phys. 45 (2000) S241 [nucl-th/0008028].

[2] B. Metsch, Eur. Phys. J. A 35 (2008) 275.

[3] W. Plessas, PoS LC 2010 (2010) 017 [arXiv:1011.0156 [hep-ph]].

[4] A. Le Yaouanc, L. Oliver, O. Pene and J. C. Raynal, Phys. Rev. D 11 (1975) 1272.

[5] T. Melde, W. Plessas and B. Sengl, Phys. Rev. D 77 (2008) 114002 [arXiv:0806.1454 [hep-ph]].

[6] S. Capstick, A. Svarc, L. Tiator, J. Gegelia, M. M. Giannini, E. Santopinto, C. Hanhart and S. Scherer et al., Eur. Phys. J. A 35 (2008) 253 [arXiv:0711.1982 [hep-ph]].

[7] A. Krassnigg, W. Schweiger and W. H. Klink, Phys. Rev. C 67 (2003) 064003 [nucl-th/0303063].

[8] T. Melde, K. Berger, L. Canton, W. Plessas and R. F. Wagenbrunn, Phys. Rev. D 76 (2007) 074020.

[9] B. Bakamjian and L. H. Thomas, Phys. Rev. 92 (1953) 1300.

[10] W. H. Klink, Phys. Rev. C 58 (1998) 3617.

[11] W. H. Klink, Nucl. Phys. A 716 (2003) 123 [nucl-th/0012031].

[12] R. Kleinhappel, Master Thesis, University of Graz (2010).

[13] T. Sato and T.-S. Lee, J. Phys. G 36 (2009) 1. 